Proceedings

\title{
Integration of Graphene on AlN Based High Frequency Resonators and Their Functionalization for Biosensing ${ }^{\dagger}$
}

Bruno Marco 1 Lorena Gordillo' José Miguel Diego Mejias ${ }^{2}$ and Enrique Iborra ${ }^{1, *}$

1 GMME-CEMDATIC, ETSI de Telecomunicación, Universidad Politécnica de Madrid, 28040 Madrid, Spain; b.marcodufort@gmail.com (B.M.); lorena.gordillo.dagallier@gmail.com (L.G.);

josemiguel.escolano@upm.es (J.M.E.); olivares@etsit.upm.es (J.O.); mclement@etsit.upm.es (M.C.)

2 Confocal Microscopy Unit, Spanish National Cancer Research Centre (CNIO), 28029 Madrid, Spain; dmegias@cnio.es

* Correspondence: eiborra@etsit.upm.es; Tel.: +34-913-367-309

† Presented at the Eurosensors 2017 Conference, Paris, France, 3-6 September 2017.

Published: 7 August 2017

\begin{abstract}
We present the successful integration of graphene on top of AlN-based solidly mounted resonators (SMRs), using a low temperature CVD process and Ni layers as catalyst. We develop a protocol to functionalize the surface of the resonators that starts with a low damage $\mathrm{O}_{2}$ radical plasma treatment of the surface of the devices that introduces a controlled density of defects on the graphene. Then, EDC/NHS chemistry is used to covalently bond streptavidin molecules to the surface of the sensors. Finally, the functionalised biosensors are assessed by analysing their response to biotinylated antibodies.
\end{abstract}

Keywords: gravimetric biosensor; graphene integration; surface functionalization; molecule detection

\section{Introduction}

Lately, there has been an increasing need for developing highly sensitive biosensors suitable for real-time detection of all type of species. Among the different types of biosensors, electroacoustic resonators based on piezoelectric materials combine the advantages of having high sensitivity, small size and electronic signal output, in addition to being label-free, and low-cost. Their operation is based on the frequency shift when a mass binds to their surface; specific binding methods for targeted species allow for selectivity. The process by which biosensors derived from high frequency resonators are fabricated is limited by the ability to functionalize their surface and by the control of the union of the molecules involved in recognizing the target. For this, graphene shows a great potential as a functionalization platform, because of its simple and compatible organic chemistry.

There are many ways to deposit graphene on top of devices, including transfer techniques of graphene obtained on other surfaces by different means and the growth of graphene directly on the final structures by chemical vapour deposition (CVD) techniques. This last is preferred for mass production being the obtained material of better quality. Usually, temperatures as high as $1000{ }^{\circ} \mathrm{C}$ are needed for CVD deposition of high quality graphene, although lower temperatures (down to $600{ }^{\circ} \mathrm{C}$ ) can be used if the appropriate catalyst and process conditions are chosen.

In this communication, we present the successful integration of graphene on top of AlN-based solidly mounted resonators (SMRs), using low temperature CVD and Ni layer as catalyst. We also describe a protocol to functionalize the surface of the resonators. Finally, the performance of the biosensors is assessed by analysing their response to streptavidin. 


\section{Materials and Methods}

\subsection{Device Fabrication}

The SMRs were fabricated by depositing Ir/AIN/Mo piezoelectric stacks on top of acoustic reflectors made up of alternating layers of low $\left(\mathrm{SiO}_{2}\right)$ and high (AlN) impedance materials (see Figure 1a). All the films were deposited by sputtering. The deposition conditions of the $\mathrm{AlN}$ and $\mathrm{SiO}_{2}$ layers in the reflector were carefully adjusted to minimize residual stresses in each film. To ensure that they withstand the high temperatures required for the graphene growth, the reflectors were subjected to heat-treatments under vacuum prior to AlN deposition, showing that the SMRs are capable of sustaining temperatures as high as $1000{ }^{\circ} \mathrm{C}$ for at least two hours, without degradation in their performance. Finally, a $100 \mathrm{~nm}$-thick catalytic nickel was thermally evaporated and patterned on the Mo top electrode for subsequent graphene deposition.
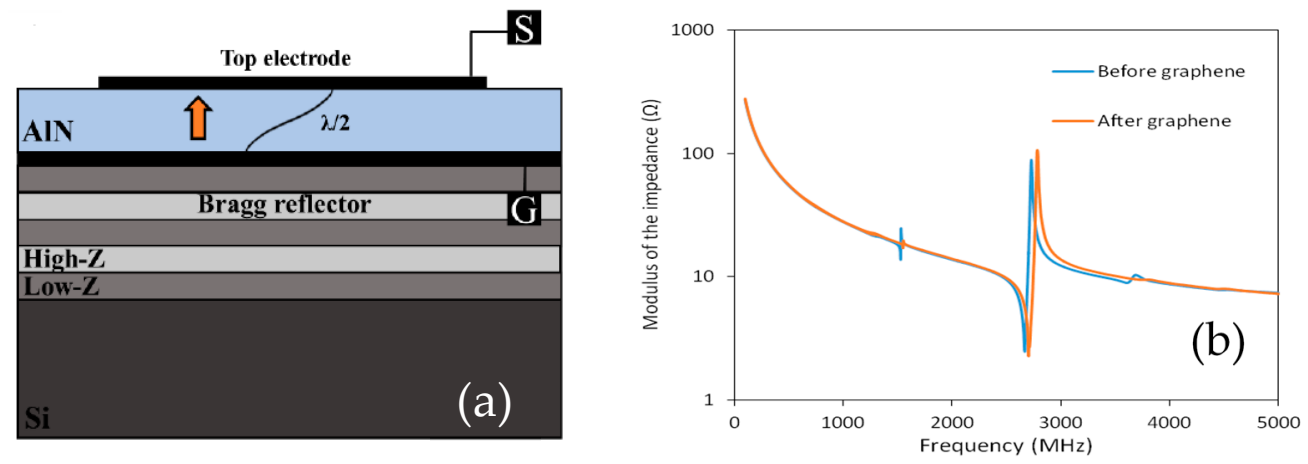

Figure 1. (a) Cross-section of a typical AlN-based SMR showing the Ir/AlN/Mo/Ni piezoelectric stack and the acoustic reflector and (b) impedance of a device before and after the growth of graphene.

\subsection{Integration of Graphene}

Graphene was grown in a custom-made cold-wall CVD on top of the Ni catalyst on the SMRs. A pyrolithic carbon heater was used as a substrate holder to decompose $\mathrm{C}_{2} \mathrm{H}_{2}$ diluted gas (acetylene) in Ar at $650{ }^{\circ} \mathrm{C}$. The total pressure in the chamber was kept constant at 130 Pa Torr. The SMRs were first heated in argon at a rate of $50{ }^{\circ} \mathrm{C} / \mathrm{min}$ up to $650{ }^{\circ} \mathrm{C}$, in order to avoid the formation of droplets in the thin Ni film [1]. They were then annealed for $4 \mathrm{~min}$ in a mixture of $\mathrm{Ar}$ and $\mathrm{C}_{2} \mathrm{H}_{2}$ (18\% in volume). Finally, the SMRs were slowly cooled down at a rate of $15^{\circ} \mathrm{C} / \mathrm{min}$ in $\mathrm{Ar}$ from $650{ }^{\circ} \mathrm{C}$ to $600{ }^{\circ} \mathrm{C}$ and then rapidly cooled to room temperature.

\subsection{Surface Functionalisation}

The first step in the functionalization process of the SMRs was to introduce chemically active oxygen containing defects on their graphene surface. For this, the samples were exposed to a lowdamage oxygen radical plasma treatment using a reactive-ion etching system. Samples were characterised before and after the plasma treatments using Raman spectroscopy in order to monitor the structural integrity of the graphene and the amount of defects generated by the plasma.

Next, streptavidin was covalently bonded to the chemically-active defects present on the surface of the SMRs using 2-step EDC/NHS protocol [2].

To assess the extent and specificity of the binding of streptavidin on the graphene we used streptavidin fluorescein isothiocyanate (FITC) conjugated; the surface was then tested by fluorescence microscopy.

\subsection{Detection}

Sensors were characterized by measuring their electrical impedance from $100 \mathrm{MHz}$ to $10 \mathrm{GHz}$ with an Agilent N5230A network analyser using calibrated RF probes. The active surface was located 
in a microfluidic system to incubate it with the binding buffer containing the target to be detected [3]. A LabVIEW application was used to accurately track the resonant frequency during the biosensing process by detecting the frequency at which the real part of the admittance peaks. This procedure allowed measuring the frequency with $1 \mathrm{kHz}$ accuracy each $3 \mathrm{~s}$.

\section{Results and Discussion}

\subsection{Integration of Graphene}

The CVD-graphene grown on top of the SMRs was characterised using Raman spectroscopy. The uniformity and quality of the coverage was assessed by obtaining multiple measurements throughout the samples. Figure 2 shows a typical Raman spectrum of as-grown graphene layer. The FWHM of the 2D peak is $40 \mathrm{~cm}^{-1}$, suggesting the presence of two to three weakly coupled layers [4]. Furthermore, the low intensity D peak, suggests that the graphene grown on the SMRs has low defect density.

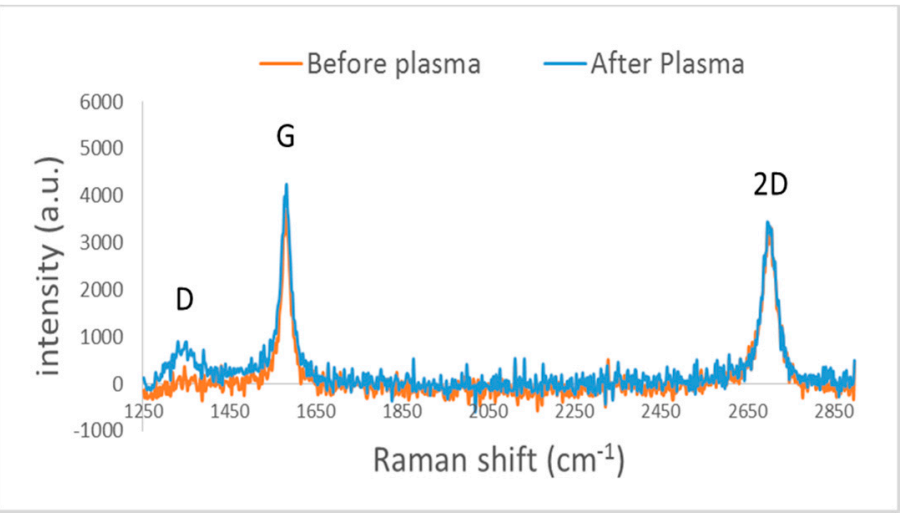

Figure 2. Raman spectra of the few-layered graphene film deposited on a SMR before and after the low damage $\mathrm{O}_{2}$ treatment. Note the appearance of the defect $\mathrm{D}$ peak after the plasma treatment.

We have analysed the influence of the process parameters (pressure, $\mathrm{Ar}-\mathrm{C}_{2} \mathrm{H}_{2}$ flow ratio and cooling rate) on obtaining few-defect and few-layer graphene. We have found that lower $\mathrm{C}_{2} \mathrm{H}_{2}$ flow rates and higher overall pressures, as well as slow variations in the total pressure, resulted in a decrease of the D peak. Additionally, slowing the cooling rate until $600{ }^{\circ} \mathrm{C}$ delivered graphene with better quality and fewer layers.

To check whether the performance of the SMRs was affected by the integration of the graphene, we measured the electrical impedance spectra of the SMRs before and after graphene deposition (see Figure 1b). The very small frequency shift suggests that the integration of the graphene does not degrade the performance of the SMRs.

\subsection{Surface Functionalisation and Bio-Detection}

After the $\mathrm{O}_{2}$ plasma treatment, the increase in the number of defects is evidenced by the rise of the D peak in the Raman spectrum (see Figure 2). Additionally, the intensities of the G and 2D peaks are unaffected by the plasma treatment. Thus, we can conclude that the plasma treatment successfully introduced intended defects in the graphene while maintaining its structural integrity.

After the plasma treatment, streptavidin was bonded to the surface of the graphene on top of the SMRs. To characterize this union, confocal fluorescent microscopy was employed. Figure 3 shows the distribution of streptavidin-FITC on top of two SMR samples covered with graphene. In the positive control (Figure 3a), the surface of the sample is uniformly covered with FITC. Almost no fluorescence is observed in the negative control (Figure 3b), where EDC/NHS was omitted from the incubation process. Since this step is essential for the formation of covalent bindings between the surface of the graphene and the streptavidin, these results show that not only was surface was successfully functionalized, but also that the union of streptavidin to the surface was specific and covalent. 
The resonator active surface area functionalized with streptavidin was incubated with rabbit polyclonal IgG (whole molecule) biotin conjugated in binding buffer. Figure 4c shows the evolution of the resonant frequency showing the effect of the binding reaction.
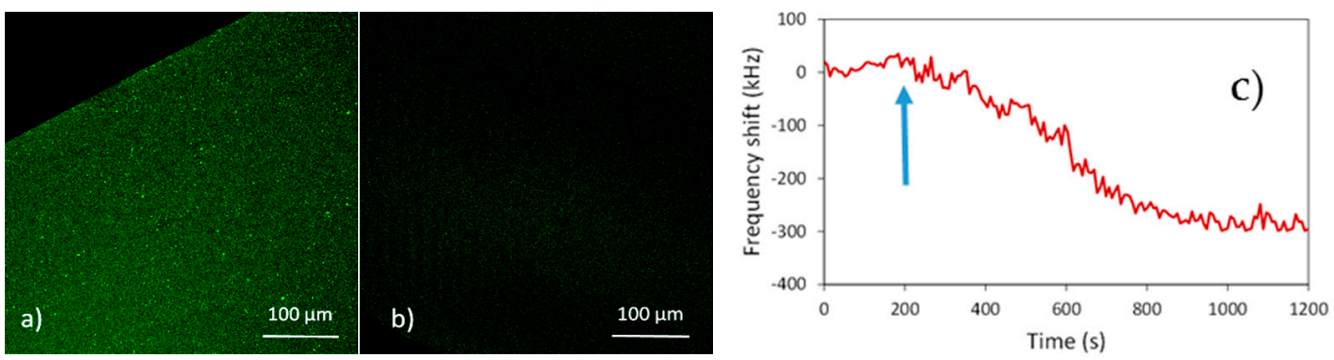

Figure 3. Confocal fluorescence microscopy images of the surface of the graphene-covered SMRs incubated with streptavidin-FITC. (a) Positive control; (b) negative control; (c) time evolution of the frequency when passing polyclonal $\mathrm{IgG}$ antibody biotin conjugated on functionalized sensors (starting time indicated by the arrow).

\section{Conclusions}

A CVD graphene deposition process was developed to grow graphene on AlN-based SMRs at low temperature, which allowed preserving the mechanical integrity of the devices. Furthermore, by successfully binding receptors to the graphene surface, a new functionalization platform for biosensing was demonstrated. To achieve surface functionalization, a low damage $\mathrm{O}_{2}$ radical plasma treatment was performed, allowing to introduce a controlled density of defects on the graphene layer. Then, EDC/NHS chemistry was used to covalently bond streptavidin molecules to the surface of the sensors. Finally, biotinylated antibodies were detected on functionalized SMRs.

Acknowledgments: This work was partially supported by the Ministerio de Economía y Competitividad del Gobierno de España through the project MAT2013-45957-R.

Conflicts of Interest: The authors declare no conflict of interest.

\section{References}

1. Golik, R.G.; Gromov, D.G.; Zhigal'skii, G.P. Analysis of Structural Transformations and Fluctuation Effects in the Nanosized Nickel Films in the Vicinity of the Melting Point. J. Commun. Technol. Electron. 2012, 57, 629-633, doi:10.1134/S1064226912030072.

2. Hermanson, G.T. Bioconjugate Techniques, 2nd ed.; Academic Press: San Diego, CA, USA, 2008.

3. DeMiguel-Ramos, M.; Díaz-Durán, B.; Escolano, J.-M.; Barba, M.; Mirea, T.; Olivares, J.; Clement, M.; Iborra, E. Gravimetric biosensor based on a $1.3 \mathrm{GHz}$ AlN shear-mode solidly mounted resonator. Sens. Actuators B Chem. 2017, 239, 1282-1288, doi:10.1016/j.snb.2016.09.079.

4. Ferrari, A.C. Raman spectroscopy of graphene and graphite: Disorder, electron-phonon coupling, doping and nonadiabatic effects. Solid State Commun. 2007, 143, 47-57, doi:10.1016/j.ssc.2007.03.052. 